\title{
La educación superior a distancia relacionada con los Objetivos de Desarrollo Sostenible. Experiencia aplicada al ODS6
}

\author{
del Teso, R. ${ }^{\text {a1 }}$, Estruch-Juan, E. ${ }^{\text {a2 }}$, Gómez, E. ${ }^{\text {a3, }}$ Soriano, J. ${ }^{\text {a4 }}$
}

aITA, Departamento de Ingeniería Hidráulica y Medio Ambiente. Escuela Técnica Superior de Ingenieros industriales. Universitat Politècnica de València.

a1 rodete@ita.upv.es, ${ }^{\text {a2 } m a e s j u a 1 @ i t a . u p v . e s, ~}{ }^{\text {a3 } e l g o s e l @ i t a . u p v . e s, ~}{ }^{a 4 j}$ jasool@ita.upv.es

\begin{abstract}
This paper analyzes the role of online higher education in achieving the Sustainable Development Goals (SDGs) proposed for 2030. It reviews the different entities that offer both courses for dissemination of the SDGs and those whose purpose is to train for their implementation. There are some limitations found in these offers such as the language of the existing curses offered, the lack of proposals of higher education, and specific trainings to implement the SDGs. This article focuses especially on the achievement of SDG6: Water and sanitation, through online higher education, with the aim of training technicians who know how to face the challenges of this SDG. The achievement of this objective, indirectly also contributes to SDGs 4, 5 and 8, as throught this teaching methodology the aim is to offer a quality and equal education, accessible from anywhere with an Internet connection, and to promote quality jobs for students.
\end{abstract}

Keywords: Sustainable Development Goals, water and sanitation, e-learning, postgraduate degree, higher education.

\footnotetext{
Resumen

En este trabajo se analiza el papel de la educación superior a distancia en la consecución de los Objetivos de Desarrollo Sostenible (ODS) planteados para 2030. Se revisan las distintas entidades que ofrecen tanto cursos de difusión de los ODS, como aquellas cuyo fin es formar para conseguir su implementación. Se localizan algunas limitaciones encontradas en dichos cursos, principalmente el idioma de la oferta formativa existente, y la falta de propuestas de educación superior, y formación especifica para implementar los ODS. El objetivo de este artículo es explicar la impartición de formación online superior como medio en la consecución del ODS6: Agua y saneamiento.

Además, esta formación contribuye a la obtención de los ODS 4, 5 y 8, ya que, a través de la metodología docente utilizada, se aspira a ofrecer una educación de calidad e igualitaria, accesible desde cualquier lugar con conexión a internet, y fomentando conseguir trabajos de calidad a los estudiantes.
}

Palabras clave: Objetivos de Desarrollo Sostenible, agua y saneamiento, formación online, posgrado, educación superior. 


\section{Introducción}

En 2015 se publicó desde las Naciones Unidas el documento "Transformando nuestro mundo: la Agenda 2030 para el Desarrollo Sostenible" (Naciones Unidas, 2015a). Un ambicioso acuerdo mundial que entró en vigor el 1 de enero de 2016 y que pretende encaminar el mundo hacia una mejora global, abordando los desafíos y retos más urgentes. Para ello se plantearon 17 Objetivos de Desarrollo Sostenible (ODS), con unas metas determinadas a cumplir en cada uno de ellos. Estos ODS son la continuación de los Objetivos de Desarrollo del Milenio (ODM) que se plantearon entre los años 2000 y 2015.

La educación queda enmarcada en diversos ODS directamente. A través del aprendizaje continuo se pretende conseguir un progreso y desarrollo social, económico y medioambiental, siendo éste clave para la implementación de los ODS. Una amplia oferta formativa es fundamental para proporcionar oportunidades de aprendizaje dinámico donde las diferentes audiencias puedan aprender a su propio ritmo y acceder al conocimiento que necesitan. Las universidades, como centros de educación superior, tienen una importante función en todo esto, proporcionando las soluciones y el conocimiento necesario para implementar los ODS, y formando personas con capacidades y habilidades profesionales específicas.

El intercambio de conocimiento es la base principal del aprendizaje. La formación a distancia, a través de un aprendizaje interactivo y en red, permite el intercambio de conocimiento desde diferentes fuentes, y consigue formar a los alumnos sin requerir desplazamiento, siendo más asequible económicamente y más sostenible, pues elimina la necesidad de desplazarse hasta los centros especializados en las diferentes tareas y campos.

\subsection{Revisión de la formación a distancia sobre ODS ofertada desde diferentes organismos internacionales}

Desde diferentes organismos internacionales se ofertan multitud de cursos relacionados con los ODS. La mayoría de estos cursos son a distancia, aunque también existen cursos presenciales impartidos por entidades, institutos o universidades. Muchas son las plataformas educativas en línea que ofrecen la posibilidad de poder cursar desde cualquier parte con acceso a Internet cursos relacionados con los ODS.

Se distinguen principalmente dos tipos de formaciones ofertadas relacionadas con los ODS. Por un lado, las encaminadas a dar a conocer y difundir los ODS, la llamada Educación para el Desarrollo Sostenible (EDS), donde se pretende dotar al estudiante de una serie de conocimientos para entender y abordar los ODS. Por otro lado, la formación encaminada a proporcionar habilidades académicas y profesionales específicas encaminadas a implementar soluciones a los ODS (SDSN Australia/Pacific, 2017).

Es necesario ofrecer oportunidades de aprendizaje que transformen el conocimiento global en un aprendizaje accesible desde cualquier lugar. Iniciativas como la del World Bank Group con su Open Learning Campus (OLC) pretenden proporcionar a las personas el conocimiento y las capacidades necesarias para la difusión y logro de los ODS (World Bank Group, 2019). Del mismo modo UNICEF y su programa AGORA con un portal de aprendizaje gratuito en 
diversas materias de cooperación y desarrollo, ofrecen oportunidades de aprendizaje para diferentes comunidades (UNICEF, 2019). También desde Naciones Unidas se han fomentado distintas campañas de aprendizaje a distancia, como el programa UNITAR (United Nations Institute for Training and Research) con el que se pretende dar a conocer cuales son las funciones de las Naciones Unidas, y muchos de los programas que llevan a cabo (Naciones Unidas, 2019). Esta formación está más enfocada a diplomáticos y funcionarios de órganos institucionales.

También desde la Red de Soluciones para un Desarrollo Sostenible (SDSN por sus siglas en inglés Sustainable Development Solution Networks) se realizan cursos online que forman parte de la SGD Academy (la academia de los ODS). Una iniciativa internacional que ofrece formación online gratuita, masiva y de calidad, impartida por expertos en desarrollo sostenible y en colaboración con más de 400 universidades de todo el mundo que forman parte de la (SDSN, 2019).

El Banco Interamericano de Desarrollo (BID) a través del Instituto Interamericano para el Desarrollo Económico y Social (INDES) promueve cursos de conocimiento abierto relacionados con el desarrollo económico y social en América Latina y el Caribe. Aunque la mayoría de los cursos son en español, también cuentan con oferta formativa en inglés, francés y portugués. Algunos de estos cursos están desarrollados en colaboración con universidades, gobiernos y otros organismos internacionales de desarrollo (BID, 2019).

Desde el Portal Educativo de las Américas impulsado por la Organización de los Estado Américanos (OEA) se ofrecen multitud de cursos y titulaciones universitarias, impartidos tanto por universidades, como por entidades públicas y privadas, ofreciendo oportunidades de formación profesional a distancia de calidad en temas claves para el desarrollo integral, siendo todos los cursos en castellano (OEA, 2019)

En materia de agua, desde la International Water Association (IWA) y su plan IWA Learn, se ofrecen diferentes cursos relacionados con el agua que son ofertados desde diferentes entidades de todo el mundo (IWA, 2019). Con ellos se pretende desarrollar las competencias necesarias para capacitar a diferentes perfiles profesionales del sector del agua.

\subsection{Limitaciones de la formación a distancia ofertada por entidades internacionales}

Una de las limitaciones que se han encontrado en la formación ofrecida es el idioma. Más de 577 millones de personas en el mundo hablan en español, incluyendo lenguas oficiales y no oficiales (Instituto Cervantes, 2018). Aproximadamente un $60 \%$ de la población latinoamericana habla este idioma, y, a excepción de España, todos los países que tienen el español como lengua oficial pertenecen a la clasificación de países con rentas reducidas realizada por el Banco Mundial (World Bank, 2019). Con estos datos, parece lógico que debiera existir una gran oferta formativa a distancia en lengua castellana que permita el acceso a cursos de capacitación y mejora educativa en países en desarrollo. En cambio, muchos de los cursos ofrecidos por entidades internacionales ofrecen la formación única y exclusivamente en inglés. 
El World Bank Group desde su Open Learning Campus ofrece más de 3500 cursos, de los cuales únicamente 50 son en español, y casi 3400 en inglés, y ninguno está ofertado por universidades (World Bank Group, 2019). En la IWA de los 139 cursos que se ofrecen, únicamente 16 son en castellano, aunque desarrollados por universidades (IWA, 2019). De los 180 cursos ofrecidos por UNICEF a través de su programa Agora, 119 son en inglés y únicamente 20 en castellano, de los cuales solamente 2 están desarrollados en colaboración con universidades (UNICEF, 2019). De los 208 eventos de formación E-learning ofertados por el programa UNITAR de las Naciones Unidas, únicamente 52 son en español (Naciones Unidas, 2019).

La mayoría de los cursos ofertados en castellano son ofrecidos por entidades internacionales de Latino América como el BID o la OEA, y una gran mayoría de estos está destinado a la difusión y solución de problemas existentes en esta parte concreta del mundo.

Junto al idioma de los cursos, se ha detectado que la mayoría de la oferta formativa existente está abierta para todos los públicos, sin exigir unos conocimientos previos necesarios. Esto por un lado permite un acceso no restrictivo a la formación, pero por otro lado condiciona los contenidos de los cursos, enfocados a todos los niveles de conocimiento y formación. De ahí que la oferta destinada a formación superior o de posgrado, y por tanto más especializada, sea minoritaria.

Además, tras la revisión de la oferta formativa existente, se ha detectado que muchos de los cursos están encaminados a la difusión y entendimiento de los ODS, siendo minoritarios los cursos destinados a la capacitación técnica para implementación directa de medidas que permitan desarrollar los ODS.

En este sentido, las universidades deben tomar las riendas de cara a formar y capacitar a las personas para implementar soluciones directas a los ODS, ofreciendo formación superior que parta de unos determinados conocimientos previos exigidos a los alumnos (SDSN Australia/Pacific, 2017). Universidades de todo el mundo deberían acogerse a estas medidas, de manera que exista una amplia oferta formativa en diferentes idiomas.

Para dar difusión a la formación a distancia ofrecida por las universidades, las grandes entidades internacionales de las que se ha hablado previamente, deberían incluir estas ofertas formativas en sus plataformas, sirviendo así como repositorio de consulta mundial de la oferta formativa ofrecida por institutos y universidades. Una oferta que incluya tanto cursos online de acceso abierto como formación superior y de posgrado a distancia, en la que se cuente con requisitos previos de acceso, como ocurre con la IWA donde existe un repositorio de cursos de posgrado.

\section{Objetivos: Los ODS en las universidades}

El objetivo general de este trabajo es promover la docencia superior a distancia impartida desde las Universidades como medio para lograr la consecución de los ODS. Las universidades como estamentos de conocimiento, investigación, innovación y desarrollo deben ser un actor fundamental para alcanzar e implementar con éxito los ODS. Deben estar a la vanguardia a la hora de encontrar soluciones técnicas que permitan lograr los ODS, 
formando a profesionales con unas cualidades específicas relacionadas con la implementación de ODS, proporcionándoles las soluciones y conocimientos necesarios para ello (SDSN Australia/Pacific, 2017).

Las diferentes entidades internacionales comentadas anteriormente tienen una amplia oferta académica destinada a todos los públicos y enfocada a comprender y difundir los ODS. Por lo que parece razonable que las Universidades y centros educativos más especializados se encarguen de ofrecer formación técnica específica. Cuanto más especializada sea esta formación mayores capacidades específicas obtendrá el alumno, por lo que se considera imprescindible incluir formación académica superior de posgrado, que permita a los profesionales y titulados universitarios adquirir competencias centradas en el cumplimiento de ODS determinados.

De esta manera se pretende que, desde las Universidades, además de ofrecer un conocimiento básico de cada uno de los 17 ODS, se adquieran habilidades y competencias clave para abordar ODS específicos, proponiendo soluciones e implementación de medidas que consigan lograr las metas definidas en cada uno de los ODS.

Por otra parte, el ODS 4 propone una educación de calidad inclusiva e igualitaria que permita promover oportunidades de aprendizaje permanente para todos (Naciones Unidas, 2019b). Para conseguir que se cumpla el ODS4, desde las Universidades es necesario ampliar las oportunidades de aprendizaje a todo tipo de público. Para ello la educación a distancia es un medio fundamental que permite formar a las personas desde cualquier parte del mundo con acceso a internet, consiguiendo una difusión de conocimiento imprescindible para lograr los ODS. Por ello es fundamental que desde las Universidades se desarrollen cursos en línea de calidad (Naciones Unidas, 2019b), que acaben con las limitaciones vistas anteriormente de la formación ofrecida por entidades internacionales. Las universidades de diferentes partes del mundo deben ofrecer cursos en línea, de calidad, en diferentes idiomas, y encaminados no únicamente a ofrecer una formación específica, sino también a adquirir titulaciones superiores, como posgrados.

\subsection{ODS6: Agua limpia y saneamiento}

Como se ha comentado anteriormente, la idea general de este trabajo es concienciar sobre la importancia de la docencia superior online impartida desde las Universidades. En concreto, el trabajo se centra en desarrollar la experiencia en la impartición de cursos y títulos de posgrado a distancia relacionados con la consecución del ODS6, utilizando una metodología y una configuración en los cursos que permite llegar a lograr otros ODS.

Uno de los ODS que se han venido tratando desde hace muchos años es la cobertura de agua y saneamiento en el mundo, y la gestión sostenible del recurso hídrico. Diferentes programas han tratado esta problemática a lo largo de los años. El programa Horizonte 2020, programa marco de investigación e innovación de la Unión Europea destinado a ofrecer ayudas económicas entre 2014 y 2020 para lograr avances y descubrimiento a nivel mundial (Comisión Europea, 2014), destinó el 35\% del presupuesto a acciones por el clima, medio ambiente, y eficiencia de recursos, haciendo especial hincapié en la gestión de recursos 
hídricos. Este programa estima que un crecimiento de tan solo el 1\% del sector del agua podría crear hasta 20.000 nuevos puestos de trabajo (Comisión Europea, 2014).

Por otro lado, de los 8 ODM definidos entre 2007 y 2015, el objetivo 7 fue garantizar la sostenibilidad ambiental. Donde la meta 7.C era reducir a la mitad, para 2015, las personas sin acceso sostenible al agua potable y a servicios básicos de saneamiento (Naciones Unidas, 2015b).

El informe de 2015 de los Objetivos de Desarrollo del Milenio indica que desde 1990 hasta 2015 se aumentó el número de personas que tienen acceso a agua por cañería, mientras que 2.100 millones de personas obtuvieron acceso a saneamiento mejorado (Naciones Unidas, 2015b). Pese a las estadísticas esperanzadoras de mejora realizadas en 2015, a día de hoy uno de los ODS más ambiciosos a cumplir es el ODS 6: garantizar la disponibilidad de agua y su gestión sostenible y el saneamiento para todos.

Unas 1.800 millones de personas en el mundo carecen de una fuente de agua potable fiable, mientras que 2.400 millones no disponen de saneamiento. Además, la escasez de agua afecta al $40 \%$ de la población mundial, siendo esta una tendencia creciente en los últimos años (Naciones Unidas, 2019c).

Tal como indica el Informe sobre el Desarrollo de los Recursos Hídricos en el Mundo de 2015 hay una aceptación universal de que el agua es un recurso natural primario esencial del que dependen casi todas las actividades sociales y económicas y los ecosistemas (WWAP, 2015). Y es que, el ODS6 es de básico cumplimiento, ya que está relacionado con muchos de los otros ODS. La prestación de un adecuado servicio de saneamiento y agua es imprescindible para lograr los ODS sobre salud e igualdad de género. Además, la gestión sostenible de recursos hídricos está relacionado directamente con el cambio climático, la conservación del medio ambiente, la producción de energía y alimentos, el crecimiento económico y el trabajo decente (Naciones Unidas, 2019c).

Tal y como sugiere el Informe de las Naciones Unidas sobre el Desarrollo de los Recursos Hídricos en el Mundo 2016: Agua y Empleo, es necesario profesionalizar el sector del agua a través de la formación del personal. Se deben ampliar los programas de estudios, ofreciendo cursos especializados para profesionales del agua. Los desafíos a los que se enfrenta el sector del agua requieren herramientas de aprendizaje innovadoras que permitan mejorar las competencias y capacidades técnicas del personal (WWAP 2016).

En perspectiva de género, es necesario incrementar la participación de las mujeres en el sector del agua, ya que actualmente solamente el $19,7 \%$ de los empleados del sector de agua y saneamiento en el mundo son mujeres, frente al $60 \%$ en el sector servicios. (Monje et al., 2016).

Todos estos datos, ponen de manifiesto la importancia de desarrollar programas formativos relacionados con la consecución del ODS6. 


\section{Desarrollo de la innovación: formación online para alcanzar las metas del ODS6}

En el sector del agua, muchos son los cursos a nivel mundial que se ofertan. Desde el grupo de investigación ITA perteneciente al Departamento de Ingeniería Hidráulica y Medio Ambiente (DIHMA) de la Universitat Politècnica de València (UPV), se imparten una serie de cursos y títulos de posgrado online sobre hidráulica, modelación de redes de agua y gestión eficiente del agua.

Los contenidos de estos cursos tienen relación directa con la implementación del ODS6, ya que permiten dotar de conocimiento a los alumnos para conseguir la mayoría de metas que tiene este objetivo de desarrollo. Pero, además, la metodología completamente online utilizada en estos cursos se enmarca directamente dentro de los ODS4, 5 y 8.

Como se ha visto, el ODS4 consiste en garantizar una educación inclusiva y equitativa de calidad y promover oportunidades de aprendizaje permanente para todos. Aunque este ODS está centrado en la enseñanza primaria y secundaria, para lograr los niveles mínimos de conocimiento en niños y adolescentes, no hay que dejar de lado la enseñanza superior, tratando de ofrecer una educación de calidad que respete la igualdad de género (ODS5) (Naciones Unidas, 2019d) y capacite a los participantes para adquirir un trabajo decente con el consecuente crecimiento económico (ODS8) (Naciones Unidas, 2019e).

Los cursos de posgrado impartidos por el ITA han sido especialmente diseñados para ser impartidos a distancia. Se utilizan recursos en formato de imagen, vídeo y texto, creados de manera lo más ilustrativa y directa posible, para tratar de evitar la falta de atención del alumno y romper con la monotonía de los cursos que utilizan siempre el mismo recurso (Guo, Kim, \& Rubin, 2014; Hsin, Cigas, \& Science, 2013).

El alumno es el encargado de desbloquear los contenidos conforme avanza en el desarrollo del curso, marcando su propio ritmo de trabajo en función de su disponibilidad temporal. A lo largo de cada una de las asignaturas se realizan diferentes evaluaciones. Las autoevaluaciones permiten que el alumno detecte posibles problemas en su aprendizaje, y los resultados obtenidos y el envío de ejercicios permiten que sea el tutor el encargado de detectar estos problemas.

El tarea del tutor es imprescindible en la consecución de los objetivos docentes del curso, siendo el encargado de fomentar la motivación de los estudiantes y de resolver cualquier duda que les pueda surgir. El tiempo de respuesta de los tutores es menor a 48 h, con la intención de los alumnos sientan la cercanía del instructor y no queden parados esperando la resolución de una duda que les impide continuar con el desarrollo del curso (Bailey y Card, 2009).

Los resultados obtenidos con la metodología utilizada son positivos, el grado de satisfacción de los estudiantes está por encima de la media de la UPV, y la tasa de abandono se mantiene por debajo de los valores medios en formación online (Confilegal, 2017).

Con la intención de facilitar el acceso a la formación superior de las comunidades más desfavorecidas, todos los cursos y títulos propios ofrecen un descuento a personas pertenecientes a países con rentas reducidas, según la clasificación del Banco Mundial, así 
como diferentes becas en colaboración con la OEA. Este tipo de descuento también es ofrecido a personas en situación de desempleo, con la intención de que una formación a distancia de calidad sea una de las posibles vías hacia el trabajo decente y el crecimiento económico de estas comunidades.

\section{Resultados}

El comienzo de la formación a distancia impartida desde el ITA fue en 2009, siendo el primer título de posgrado impartido en 2012. Desde entonces se han creado 23 cursos que se reparten en 5 titulaciones de posgrado diferentes. Este crecimiento en los cursos ha venido favorecido por la necesidad y demanda de cursos técnicos que permitan capacitar al personal en materias relacionadas con el agua.

\subsection{Evolución de contenidos y alumnos de América Latina}

Junto al crecimiento de la oferta formativa, se ha experimentado un aumento de alumnos pertenecientes a países en desarrollo, en la Figura 1 se observa una comparación entre la oferta de cursos realizada desde 2013 y la matriculación de alumnos de América Latina.

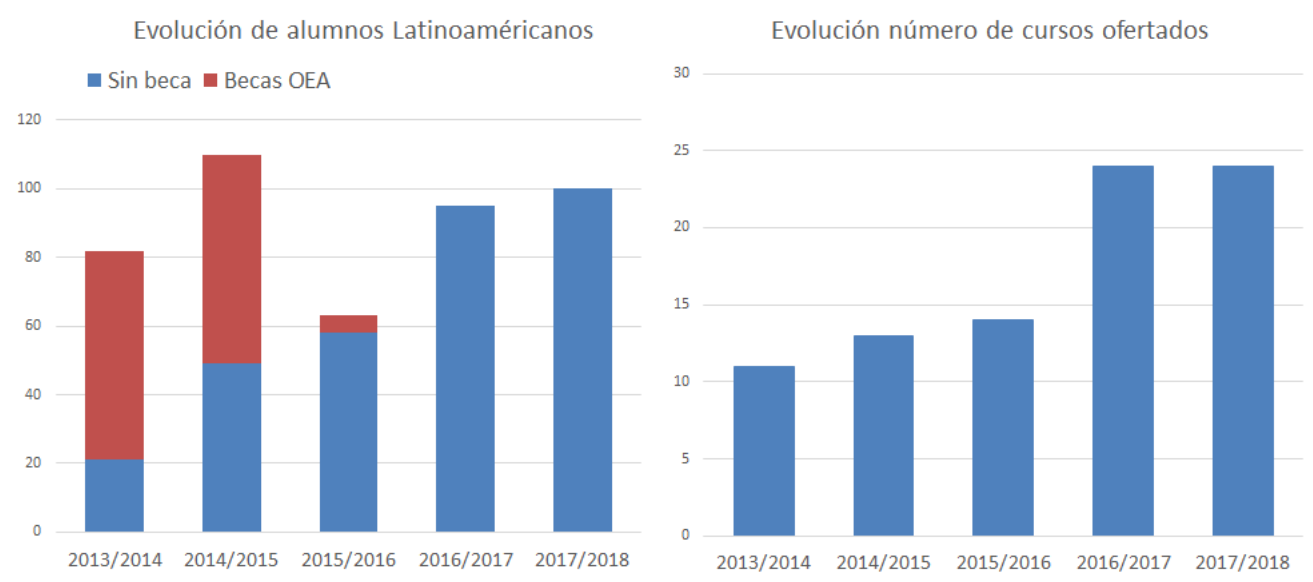

Fig. 1 Comparación entre la oferta de cursos y la evolución de alumnus latinoaméricanos.

Cabe destacar el pico de alumnos de los cursos 2013/2014 y 2014/2015, marcado en rojo en el gráfico, correspondiente a alumnos matriculados a través del programa de becas ofrecido por la Organización de los Estados Americanos (OEA), y las cuales ya no se ofertan desde 2016.

El $41 \%$ de los alumnos matriculados en los títulos de posgrado ofertados por el ITA desde 2014 proviene de España, mientras que el 59\% restante proviene de países latinoamericanos (Figura 2). El país de Latino América con más alumnos es Colombia (28\% del total de alumnos latinoamericanos). Una de las grandes medidas de este país es disminuir el Índice de Pobreza Multidimensional (IPM), el cual incluye el acceso a servicios públicos de agua y saneamiento. Diferentes medidas e inversiones se han realizado para alcanzar en 2017 una cobertura de agua del $92,4 \%$ y de saneamiento del $88,2 \%$, siendo la meta establecida para 
2030 cubrir el $100 \%$ de ambos servicios, tanto en zona rural como en zona urbana, donde la cobertura actual no llega al 74\% (Gobierno de Colombia, 2018).

El segundo país con más número de alumnos es Uruguay (12\% del total de alumnos latinoamericanos) donde ocurre algo similar a Colombia. En 2016 el 95,2 \% de su población tenía acceso a agua segura y se busca llegar al $100 \%$ en 2030. En cambio, están algo lejos en cuestión de saneamiento donde la gestión segura de este servicio en 2016 únicamente fue del 45,2\% siendo el objetivo en 2030 de llegar al 100\% (Presidencia República Oriental del Uruguay, 2018). Para lograr esta meta se ha desarrollado un Plan Nacional de Aguas (PNA) con diferentes medidas políticas, y un apartado exclusivo dedicado a la educación e investigación en gestión del agua, donde se habla de la formación y capacitación de técnicos y profesionales especializados. (MVOTMA, 2017). Quizá estas metas y políticas llevadas a cabo se han visto reflejadas en un aumento del número de alumnos provenientes de estos países que buscan profesionalizarse en un sector como es el del agua y saneamiento.
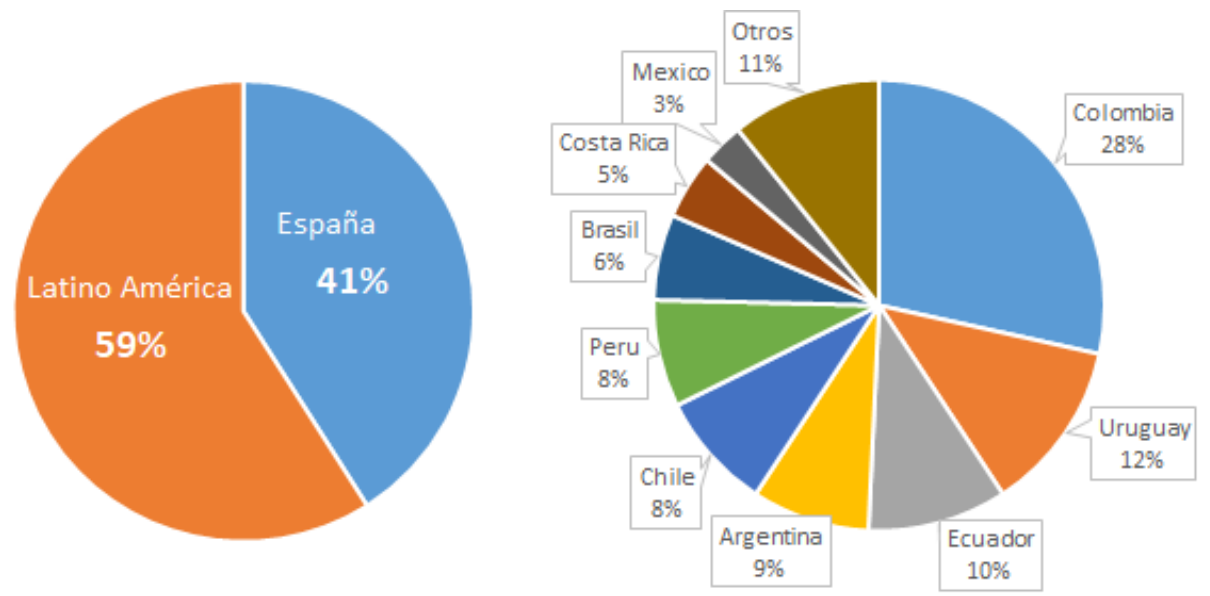

Fig.2 Nacionalidad de los alumnus matriculados en titulos de posgrado ofertados por el ITA

No solamente la metodología online utilizada favorece el acceso a alumnos de diferentes partes del mundo. La estructura formativa creada permite acceder a las asignaturas que componen los títulos de posgrado de manera individual, para posteriormente poder ser convalidadas de cara a obtener una titulación de posgrado. Esto posibilita a corto plazo adquirir unos conocimientos determinados de asignaturas puntuales, y a largo plazo el acceso a titulaciones de posgrado que ofrecen una formación más amplia, permitiendo una flexibilidad en cuanto a la gestión de la formación del estudiante.

De todos los alumnos que realizaron alguna asignatura de manera individual durante el curso 2017/2018 (130 en total), 37 ya habían cursado algún módulo anteriormente, es decir, casi el $30 \%$ de los alumnos había participado en alguna otra ocasión en las acciones formativas impartidas por el ITA. Además, de los 89 alumnos matriculados desde 2017 en alguno de los títulos de posgrado, 24 ya habían cursado previamente algún módulo individual o título, por tanto, el $27 \%$ de los alumnos que realizaron un título de posgrado entre 2017 y 2019, convalidaron asignaturas realizadas individualmente en cursos previos. 
Otra de las acciones llevadas a cabo para la mejora del empleo y el crecimiento económico (ODS8) es la gestión del apostillado de La Haya de los títulos para que las titulaciones de posgrado surtan efecto en el extranjero y sirva como vía para el acceso a trabajos cualificados y de calidad. Los títulos se envían directamente apostillados, sin necesidad de desplazamientos por parte de los alumnos.

La formación de posgrado más completa impartida por el ITA es el Máster en Gestión Eficiente del Agua Urbana, el cual cuenta con 13 asignaturas en su fase lectiva. Una vez concluida la fase lectiva, los alumnos tienen un año para realizar un Trabajo Final de Máster (TFM). Los tutores del curso proponen líneas de interés que pueden guiar a los alumnos hacia qué tipo de proyecto desarrollar, pero nunca se proponen trabajos específicos. Con esto se busca que sea el alumno el que encuentre un problema en su entorno que pueda resolver con los conocimientos adquiridos en el Máster. De esta manera se han desarrollado trabajos encaminados a solucionar problemas en abastecimientos de agua con consumos intermitentes, para disponer de agua las 24 horas del día. Mejorar redes de distribución y saneamiento de agua, o realizar políticas de gestión de agua encaminadas a la reducción de fugas y pérdidas del recurso hídrico, entre muchos otros.

La gran participación de alumnos extranjeros en la oferta formativa de cursos y títulos de posgrado se debe a la metodología completamente online utilizada, incluso para las defensas del TFM, las cuales se realizan a través de videoconferencia, lo que permite ahorrar los costes de desplazamiento, favoreciendo la sostenibilidad del medio.

\subsection{Formación técnica destinada a empleados de España}

Según el "XIV Estudio Nacional de Suministro de Agua Potable y Saneamiento en España 2016”, más de 26.000 empleos directos se generan en España con el sector del agua urbana (abastecimiento, saneamiento y depuración), siendo el 93\% de estos empleos fijo. Aunque únicamente el 17\% del personal es titulado superior o grado medio (AEAS-AGA, 2016).

Estos datos reflejan la necesidad por parte de las empresas de ofrecer una formación continua a sus empleados, de ahí que cada vez existan más convenios de formación entre empresas y universidades (Aqualia, 2019).

Cada vez son más las empresas que apuestan por la formación a distancia de sus empleados, siendo esta metodología compatible con el volumen de trabajo existente. Una formación a través de plataformas e-learning que ofrecen acceso 24 horas al día, los 365 días al año, de manera que los participantes puedan gestionar el tiempo de dedicación en función de su disponibilidad personal y laboral, adaptando el estudio a los periodos con menor carga laboral. Así lo demuestra las estadísticas sobre formación a empleados en empresas de abastecimiento y saneamiento en España, donde se indica que más del $97 \%$ de los empleados reciben formación al año (AEAS-AGA, 2018). En la Figura 3 se puede ver que los empleados que mayor formación reciben son los titulados superiores: 

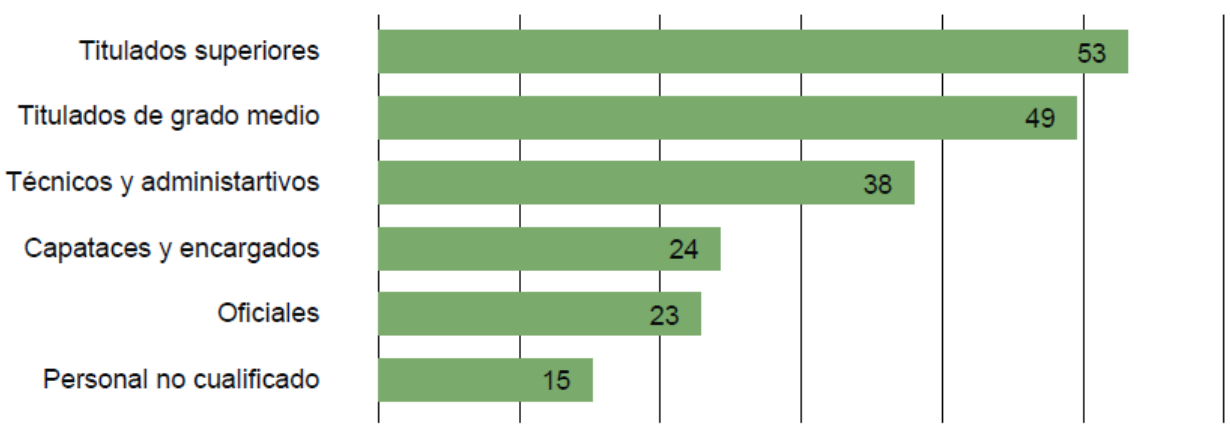

Fig.3 Número de horas de formación al año por tipo de empleado (Fuente: AEAS-AGA, 2018)

La formación a distancia es una metodología que permite actualizar y renovar los conocimientos de los empleados en las distintas áreas de trabajo, esto influye directamente en el desarrollo profesional del trabajador y por ende en la propia empresa en la que labora.

En España, a través de la Fundación Estatal para el Empleo (FUNDAE), se ofrece la posibilidad de bonificar el coste de los cursos realizados por empleados pertenecientes a empresas. La oferta formativa del ITA es bonificable a través de FUNDAE, lo que ha permitido capacitar desde 2012 a más de 300 alumnos en el sector del agua. De los alumnos que realizan asignaturas de manera individual aproximadamente el $50 \%$ provienen de empresas que solicitan la bonificación de la formación realizada. Y es que, tal como se propone desde el ODS4 las políticas sobre educación son una pieza clave para el desarrollo, por lo que la oportunidad de tener becas que permitan el acceso a una educación superior es una garantía de mejora de las condiciones de la población.

\section{Conclusiones}

Se han detectado una serie de limitaciones en la oferta formativa a distancia ofrecida por organizaciones internacionales. El lenguaje de los cursos a distancia ofrecidos exclusivamente en inglés dificulta el acceso a los hablantes de lengua hispana. Además, muchos de los cursos son de acceso abierto y no requieren conocimientos previos para realizarlos, ni están desarrollados por universidades, por lo que no ofrecen capacitaciones técnicas específicas ni titulaciones de posgrado, sino que es accesible a todo el mundo sin cualificación necesaria.

Las universidades, con su amplia oferta formativa (enseñanzas de grado y posgrado, títulos propios, formación específica presencial y a distancia, convenios formativos con empresas prácticas, enseñanza a distancia...), son una pieza clave en la implementación de los ODS, ya que desde las universidades se puede proporcionar una serie de conocimientos y capacidades en materias relacionadas con los ODS.

El ODS6 está directamente relacionado con otros objetivos. Concretamente, el agua está relacionada con muchos aspectos del crecimiento económico sostenido, inclusivo y sostenible, el empleo pleno y productivo y el trabajo digno para todos. 
Un ejemplo de cómo las universidades pueden ayudar a lograr los ODS son los cursos de posgrados online impartidos por el ITA, donde se ofrece una formación destinada a adquirir capacidades concretas relacionadas con el agua, lo que facilita en gran medida el cumplimiento directos del ODS6, pero no únicamente este objetivo de desarrollo sino otros muchos como el ODS 4, el ODS5 y el ODS8 al tratar de ofrecer una educación de la máxima calidad posible accesible desde cualquier parte del mundo con conexión a internet que permita conseguir trabajos de calidad a los estudiantes. Con la formación ofrecida por el ITA se pretende dar una formación técnica de calidad, que contribuya al aumento de los servicios de agua y saneamiento gestionados de manera segura y eficiente en todos los países.

La tecnología está cambiando el panorama del aprendizaje, multitud de plataformas tartan de ofrecer un aprendizaje accessible e intuitive al estudiante, combinando herramientas como documentos, vídeos o foros y chats que promueven el intercambio continuo entre participantes, todas encaminadas a ofrecer un plan de aprendizaje lo más completo posible.

\section{Referencias}

AEAS-AGA (2016). XIV Estudio Nacional de Suministro de Agua Potable y Saneamiento en España 2016. Asociación Española de Abastecimientos de Agua y Saneamiento (AEAS) - Asociación Española de Empresas Gestoras de los Servicios de Agua Urbana (AGA).

AEAS-AGA (2018). XV Estudio Nacional de Suministro de Agua Potable y Saneamiento en España 2018. Asociación Española de Abastecimientos de Agua y Saneamiento (AEAS) - Asociación Española de Empresas Gestoras de los Servicios de Agua Urbana (AGA).

Aqualia (2019). Información Real del Agua. <http://www.informacionrealdelagua.com/> [Consulta: 23 de marzo de 2019]

BAILEY, C. J., CARD, K. A. (2009). "Effective pedagogical practices for online teaching: Perception of experienced instructors". The Internet and Higher Education, 12(3-4), 152-155.

BID (2019). Banco Interamericano de Desarrollo. BID Mejorando vidas. Aprendizaje abierto para

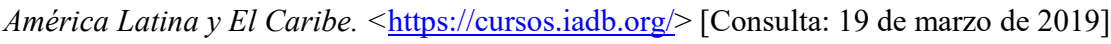

Comisión Europea (2014). HORIZON 2020 en breve. El Programa Marco de Investigación e Innovación de la Unión Europea. Luxemburgo: Oficina de Publicaciones de la Unión Europea.

Confilegal. (2017, June 14). La formación “on line” no funciona del todo bien: $30 \%$ de abandono en los cursos de pago y $90 \%$ en los gratuitos. $<$ https://confilegal.com/20170614-la-formacion-on-line-nofunciona-del-bien-30-abandono-los-cursos-pago-90-los-gratuitos/> [Consulta: 29 de mayo de 2019]

Gobierno de Colombia (2018). Reporte Nacional Voluntario Colombia 2018. Departamento Nacional de Planeación. Bogotá D.C., Colombia.

GUO, P. J., KIM, J., RUBIN, R. (2014). "How Video Production Affects Student Engagement:An Empirical Study of MOOC Videos". In L@S '14 Proceedings of the first ACM conference on Learning @ scale conference (pp. 41-50). Atlanta, Georgia, USA. http://doi.org/10.1145/2556325.2566239

HSIN, W., CIGAS, J., SCIENCE, C. (2013). "Short Videos Improve Student Learning in Online". Journal of Computing Sciences in College, 28(5), 253-259. 
Instituto Cervantes (2018). El español: una lengua viva. Informe 2018. Departamento de Comunicación Digital del Instituto Cervantes.

IWA (2019). International Water Association. IWA Learn. The place for learning \& professional development in the Water Sector. $<$ http://www.iwa-network.org> [Consulta: 20 de marzo de 2019]

MONJE, A. NUÑEZ, A. SUBIZA, D. (2016). "¿Tiene género el agua?". Banco Interamericano de Desarrollo.

MVOTMA Ministerio de Vivienda Ordenación Territorial y Medio Ambiente (2017). Plan Nacional de Aguas. Montevideo, Uruguay.

Naciones Unidas (2015a) Transformar nuestro mundo: la Agenda 2030 para el Desarrollo Sostenible, A/RES/70/1 Resolución aprobada por la Asamblea General el 25 de septiembre de 2015.

Naciones Unidas (2015b) Objetivos de Desarrollo del Milenio. Informe 2015. Nueva York: Naciones Unidas.

Naciones Unidas (2019a). UNITAR. United Nations Institute for Training and Research.

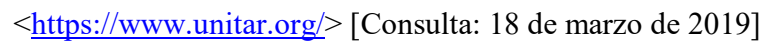

Naciones Unidas (2019b). ODS. Objetivo 4: Garantizar una educación inclusiva, equitativa y de calidad y promover oportunidades de aprendizaje durante toda la vida para todos. $<$ https://www.un.org/sustainabledevelopment/es/education/> [Consulta: 8 de marzo de 2019]

Naciones Unidas (2019c). ODS. Objetivo 6: Garantizar la disponibilidad de agua y su gestión sostenible y el saneamiento para todos. < https://www.un.org/sustainabledevelopment/es/water-and-

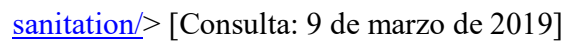

Naciones Unidas (2019d). ODS. Objetivo 5: Lograr la igualdad entre los géneros y empoderar a todas las mujeres y las niñas. < https://www.un.org/sustainabledevelopment/es/gender-equality/> [Consulta: 9 de marzo de 2019]

Naciones Unidas (2019e). ODS. Objetivo 8: Promover el crecimiento económico sostenido, inclusivo $y$ sostenible, el empleo pleno $y$ productivo $y$ el trabajo decente para todos.

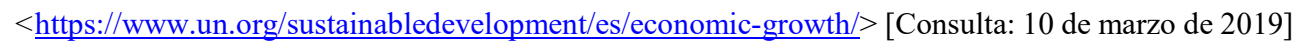

OEA (2019). Organización de los Estados Américanos. Portal Educativo de las Américas. $<$ http://www.educoas.org $>$ [Consulta: 20 de marzo de 2019]

Presidencia República Oriental del Uruguay (2018). Informe Nacional Voluntario Uruguay 2018. Uruguay.

SDSN Australia/Pacific (2017): Getting started with the SDGs in universities: A guide for universities, higher education institutions, and the academic sector. Melbourne: Sustainable Development Solutions Network-Australia/Pacific.

SDSN (2019). Sustainable Development Solutions Network. A global initiative for the United Nations. SDG Academy < https://sdgacademy.org/courses/> [Consulta: 20 de marzo de 2019]

UNICEF (2019). AGORA. El centro global de aprendizaje y desarrollo de UNICEF. $<$ https://agora.unicef.org/> [Consulta: 18 de marzo de 2019]

World Bank (2019). World Bank Country and Lending Groups. $<$ https://datahelpdesk.worldbank.org/> [Consulta: 24 de marzo de 2019]

World Bank Group (2019). Open Learning Campus. Accelerating solutions through learning. $<$ https://olc.worldbank.org/> [Consulta: 18 de marzo de 2019] 
La educación superior a distancia relacionada con los Objetivos de Desarrollo Sostenible. Experiencia aplicada al ODS6

WWAP United Nations World Water Assessment Programme (2015). The United Nations World Water Development Report 2015. Water for a sustainable World. París, UNESCO.

WWAP Programa Mundial de Evaluación de los Recursos Hídricos de las Naciones Unidas (2016). Informe de las Naciones Unidas sobre el Desarrollo de los Recursos Hidricos en el Mundo 2016: Agua y Empleo. París, UNESCO.

(c)) BY-NC-ND 2019, Universitat Politècnica de València 